\title{
Los valores sociales compartidos por los adolescentes melillenses
}

\author{
María Tomé Fernández, Universidad de Granada, España \\ Jorge Expósito López, Universidad de Granada, España \\ Emilio Berrocal de Luna, Universidad de Granada, España
}

\begin{abstract}
Resumen: Este estudio tiene como objetivo conocer los valores sociales que comparten los adolescentes de la ciudad autónoma de Melilla. Para ello, se ha seleccionado una muestra de 208 estudiantes, de distintos orígenes culturales, pertenecientes a diferentes centros escolares de la ciudad. Tras evaluarlos, utilizando los valores sociales representados en el test de valores de Casares (1995), obtuvimos que todo el alumnado seleccionado reaccionan con agrado a los valores de amabilidad, asociación, bien común, civismo, colaborar, conversar, convivencia, derechos, diálogo, escuchar, estado, hospitalidad, igualdad de oportunidades, leyes, pluralismo, política, relacionarse, respeto, reunión, sociable, sociedad, solidario, tolerante y valor. Sólo se han encontrado diferencias significativas en la valoración del valor constitución.
\end{abstract}

Palabras Clave: Valores Sociales, Adolescentes, Convivencia Intercultural, Test de Valores

Abstract: This study aims to understand the social values shared by teenager's students in the autonomous city of Melilla. On this way, a randomized sample of 208 students from different schools and different cultural provenance was selected. After their evaluation, using social values considered on a standard values test by Casares (1995), the following outcomes were founded. The whole sample of students reacted with pleasure to values such as kindness, partnership, common good, citizenship, collaborate, chat, coexistence, rights, dialogue, listen, mood, entertainment, equal opportunity, laws, pluralism, politics, relationships, respect, meeting, social, society, solidarity, tolerance and courage. Only on the value of constitution significant differences were founded.

Keywords: Social Values, Adolescents, Intercultural Coexistence, Test Values

\section{Introducción}

$\mathrm{E}$

N LA SOCIEDAD actual nos encontramos ante una realidad en constante cambio, formada por individuos muy distintos con diferentes culturas, etnias o religiones. Este hecho ha fundamentado la convivencia intercultural en muchas ciudades occidentales, entre la que se encuentra la ciudad autónoma de Melilla.

No obstante, lejos de tratarlo como algo problemático, Bilbeny (2002) recomienda la convivencia intercultural, asegurando que este tipo de convivencia proporciona ventajas al ciudadano independientemente del origen cultural al que pertenezca. Según este autor por un lado nos ayuda a conocer las identidades de otros individuos y por otro lado nos facilita el conocimiento de nuestra propia identidad. Además, para otros autores esta convivencia

Revista Internacional de Educaci ón y Aprendizaje

Volumen 1, 2013, http://sobrelaeducacion.com/revistas/coleccion/, ISSN 2255-453X

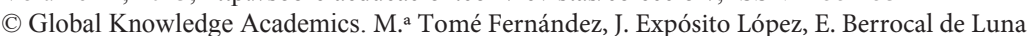

Todos los Derechos Reservados. Permisos: soporte@gkacademics.com

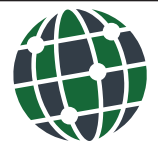


garantiza modos de comunicación, intercambios de valores y conexiones entre miembros de diferentes grupos culturales (Ruiz, 2002; Sabariego, 2002; Buendía, 2007).

Sin embargo, debemos tener en cuenta que los valores que se comparten en la convivencia intercultural pueden estar influidos por el grado de religiosidad que presenten las personas (Canetti-Nisim, 2004). Tanto es así, que desde hace décadas los psicólogos de la religión estudian los puntos conflictivos entre la fuerte convicción religiosa y la adquisición de valores (Hunsberger, 1995; Schwartz \& Huismans, 1995).

Existen investigaciones que demuestran que la religiosidad influye negativamente en la adopción de valores, llegando incluso a ser un obstáculo que dificulta su aprendizaje y socialización (Bellah, 1976; Lipset, 1981; Stark, 2001) y algunos autores consideran que hay tensiones entre la tradición cultural y religiosa y la adquisición de los derechos humanos y por tanto, de los valores que sustentan estos derechos (Gearon \& Brown, 2003). Estas discrepancias entre convicción religiosa y aceptación de valores sociales comunes, podrían entorpecer la convivencia intercultural, ya que tal y como afirman algunos autores (BeitHallahmi, 2001; Canetti-Nisim, 2004), los ciudadanos de fuertes creencias religiosas son más conflictivos en la convivencia, alcanzando en algunas ocasiones, la violencia para promover sus creencias y suprimir las de los otros grupos culturales. A esta línea se unen las aportaciones que demuestran que el valor de tolerancia está más arraigado en aquellos ciudadanos que se consideran no creyentes (Stouffer, 1955; Herek, 1987; Sullivan \& Transue, 1999) y las investigaciones que determinan que a medida que la religiosidad del individuo se asocia al autoritarismo, esta fomentará la adopción de antivalores como los de racismo y homofobia (Wylie \& Forest, 1992; Laythe, Finkel, \& Kirkpatrick, 2001).

Por otro lado, existen investigaciones en las que se afirman totalmente lo contrario (Kohlberg, 1974; Kohlberg \& Power, 1981), y consideran la religiosidad de la persona como una predisposición positiva para la adquisición y socialización de valores que fomentan la convivencia. Estos estudios consideran que las personas con convicción religiosa tienen seguridad y sentido vital suficiente para hacer un buen juicio y una elección moral adecuada en situaciones de convivencia. Haldane (1986) relaciona el cristianismo con la transmisión de los valores de libertad, igualdad y responsabilidad social, y por ello considera que los individuos de esta religión convivirán dentro de los principios de justicia social y democracia. Además, siguiendo en esta línea, en un estudio de Pérez-Delgado (1992) se demostró que las personas religiosas, a diferencia de las no creyentes, prefieren los valores relacionados con el respeto, la igualdad, la indulgencia o la servicialidad.

Centrándonos en la investigaciones realizadas con adolescentes, la mayoría de los estudios que tenían por objetivo conocer la influencia de la religiosidad en la adquisición de valores, se han hecho desde el análisis de antivalores democráticos como los relacionados con la delincuencia (Benson, 1993) y tan sólo se asemejan al estudio de los valores sociales en adolescentes, aquellas investigaciones centradas en conocer la influencia de la religiosidad en las conductas prosociales de los jóvenes y en la utilización de los valores que estas conductas conllevan (Hardy, Padilla-Walkera \& Carlo, 2008). En estas últimas investigaciones se asegura que la religiosidad influye positivamente en el comportamiento prosocial del joven, porque permite una puesta en práctica de algunos valores. Estos estudios hacen una especial mención al valor de ayuda (Batson et al., 1979; Ritzema, 1979; Bernt, 1989; Ellison, 1992; Donahue \& Benson, 1995; Wilson \& Janoski, 1995; Youniss, McLellan, \& Yates, 1999). La adquisición de valores en esta población, debe verse como un proceso en el que el joven acepta primeramente el valor y posteriormente, lo integran totalmente en su yo in- 
terno, de tal manera que el comportamiento externo quedará controlado por el valor previamente interiorizado (Grusec \& Goodnow, 1994; Grolnick, Deci, \& Ryan, 1997; Grusec, 2002). En estos casos, el valor aprendido en la adolescencia, se vinculará con la personalidad futura del joven (Yik \& Tang, 1996).

A pesar de las discusiones teóricas existentes acerca de la influencia de la religiosidad en la adquisición de valores, las investigaciones empíricas que se centran en conocer los valores sociales que comparten los adolescentes de diferentes religiones, son escasas y aún más en el contexto que nosotros la realizamos, de ahí la importancia de nuestra investigación.

\section{Método}

\section{Participantes}

La población de este estudio la forman todos los adolecentes melillenses escolarizados en algunos de los centros de Educación Secundaria Obligatoria. Para la obtención de la muestra, nos servimos de aquellos estudiantes, que previa autorización paterna, quisieron participar de forma voluntaria.

La muestra quedó constituida por 208 adolescentes melillenses de edades comprendidas entre los 13 y los 17 años. El 10,6\% de la muestra tiene 13 años, el 40,4\% 14 años, el 38,5\% 15 años, el 8,7\% 16 años, y el 1,9\% 17 años. Además, el 51,4\% de los adolescentes son de género masculino y el $48,6 \%$ son de género femenino.

Por otro lado, la distribución por religiones de la muestra es la siguiente: el 8,7\% profesan la religión católica, el $68,3 \%$ la religión islámica y el $23,1 \%$ no se considera de ninguna religión, tal y como se muestra el gráfico 1.

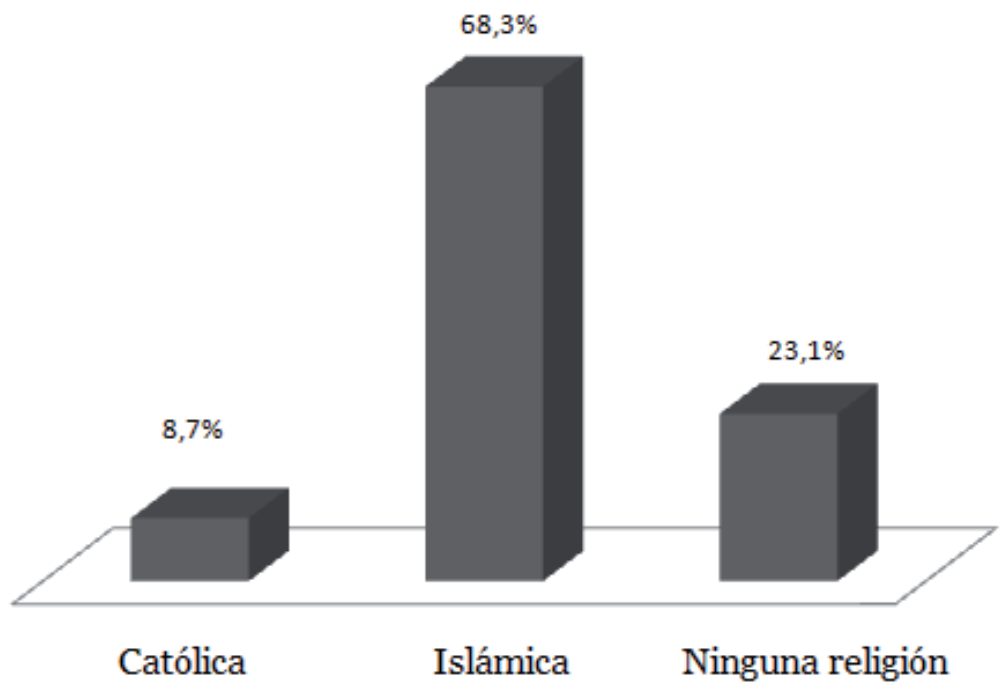

Gráfico 1: Distribución por religiones de la muestra 


\section{Problema, hipótesis y variables de la investigación}

El problema de investigación lo concretamos en la siguiente pregunta: ¿que respuestas dan los jóvenes melillenses a los valores sociales presentados y cuáles de las respuestas positivas dadas son compartidas por el alumnado de diferentes religiones y/o de ninguna religión?

Ante este problema de investigación, planteamos como hipótesis de partida que el alumnado que profesa diferentes religiones o ninguna, presentarán también respuestas diferentes ante los valores sociales evaluados.

En este estudio, se tomará como variable independiente la variable religión, que se presenta con diferentes niveles: religión católica, islámica y de ninguna religión. Y como variables dependientes todos los valores sociales que se analizan a partir del cuestionario de Casares (1995) (amabilidad, asociación, bien común, civismo, colaborar, constitución, conversar, convivencia, derechos, diálogo, escuchar, estado, hospitalidad, igualdad de oportunidades, leyes, pluralismo, política, relacionarse, respeto, reunión, sociable, sociedad, solidario, tolerante y valor).

\section{Diseño}

Para analizar los valores sociales que comparten los adolescentes melillenses, se ha realizado un estudio transversal, en el que se ha llevado a cabo una comparación intragrupo de las diferentes creencias que componen la muestra.

\section{Instrumento para la recogida de información}

El instrumento utilizado en esta investigación es una adaptación del test de valores elaborado por Casares (1995), cuya validez y fiabilidad se muestran en el trabajo realizado por dicha autora y cuyo contexto no difiere del presentado en este estudio.

El cuestionario inicial consta de 250 ítems distribuidos en 14 dimensiones. Estas dimensiones son extraídas de la clasificación axiológica de valores realizada por Gervilla (1991) y están formadas por los valores corporales, afectivos, intelectuales, individuales, liberadores, morales, volitivos, sociales, ecológicos e instrumentales.

Para nuestro estudio hemos extraído sólo los valores sociales que pueden verse en la tabla que se adjunta (Tabla1). 
Tabla 1: Cuestionario de Valores Sociales

\begin{tabular}{|c|c|c|c|c|c|}
\hline Palabra & MA & A & I & $\mathrm{D}$ & MD \\
\hline \multicolumn{6}{|c|}{ Amabilidad } \\
\hline \multicolumn{6}{|c|}{ Asociación } \\
\hline \multicolumn{6}{|c|}{ Bien común } \\
\hline \multicolumn{6}{|l|}{ Civismo } \\
\hline \multicolumn{6}{|l|}{ Colaborar } \\
\hline \multicolumn{6}{|c|}{ Constitución } \\
\hline \multicolumn{6}{|c|}{ Conversar } \\
\hline \multicolumn{6}{|c|}{ Convivencia } \\
\hline \multicolumn{6}{|l|}{ Derechos } \\
\hline \multicolumn{6}{|l|}{ Diálogo } \\
\hline \multicolumn{6}{|l|}{ Escuchar } \\
\hline \multicolumn{6}{|l|}{ Estado } \\
\hline \multicolumn{6}{|c|}{ Hospitalidad } \\
\hline \multicolumn{6}{|c|}{ Igualdad de Oportunidades } \\
\hline \multicolumn{6}{|l|}{ Leyes } \\
\hline \multicolumn{6}{|c|}{ Pluralismo } \\
\hline \multicolumn{6}{|l|}{ Política } \\
\hline \multicolumn{6}{|c|}{ Relacionarse } \\
\hline \multicolumn{6}{|l|}{ Respeto } \\
\hline \multicolumn{6}{|l|}{ Reunión } \\
\hline \multicolumn{6}{|l|}{ Sociable } \\
\hline \multicolumn{6}{|l|}{ Sociedad } \\
\hline \multicolumn{6}{|l|}{ Solidario } \\
\hline \multicolumn{6}{|l|}{ Tolerante } \\
\hline Valor & & & & & \\
\hline
\end{tabular}

Como se puede observar, al alumnado se le presenta una escala tipo likert, en la que tienen que marcar con una $\mathrm{x}$ la sensación que le produce dicha palabra. Las respuestas oscilan desde la sensación de $\mathrm{MA}=$ muy agradable a $\mathrm{MD}=$ muy desagradable, pasando por $\mathrm{A}=$ agradable, $\mathrm{I}=$ indiferente $\mathrm{y} \mathrm{D}=$ desagradable.

El valor de fiabilidad, entendida esta como la consistencia interna fue: $\alpha($ Cronbach $)=$ 0,98. La validez realizada por consenso de diez jueces, se obtuvo de la valoración de los ítems del cuestionario a través de una escala tipo likert con cinco opciones de respuestas. 
Fueron eliminados cuatro ítems que obtuvieron puntuaciones por debajo de tres en dicha escala.

\section{Procedimientos para la recogida de información}

En primer lugar, pedimos el consentimiento de las autoridades educativas de la ciudad autónoma. Tras la resolución positiva de estas, se informó a los directores de los centros. Aquellos que eran favorables a colaborar, nos citaron en diferentes días para realizar la pasación del cuestionario en las distintas aulas.

Finalmente, participaron todos los centros de Educación Secundaria de la ciudad excepto uno.

\section{Análisis de los resultados}

Para la realización del análisis estadístico del estudio, se ha utilizado el software SPSS en su versión 17.0.

Para comprobar la existencia de diferencias significativas entre las respuestas de los diferentes grupos religiosos, se empleó el estadístico de contraste no paramétrico KruskallWallis para muestras independientes, ya que los datos no se ajustan a la distribución normal.

Al realizar dicho análisis, se observa que no hay diferencias significativas entre las respuestas dadas a los valores sociales de los diferentes grupos religiosos, excepto en el valor social de constitución $(a=0.03)$ (Ver tabla 2). 
Tabla 2: Prueba de Kruskall-Wallis para muestras independientes para las variables Valores Sociales y Creencia Religiosa

\begin{tabular}{|l|c|c|c|c|}
\hline \multirow{2}{*}{ Valor } & \multirow{2}{*}{ Significación } & \multicolumn{3}{|c|}{ Religión y grado de aceptación } \\
\cline { 3 - 5 } & & Cristianos & Islámicos & Ninguna religión \\
\hline Amabilidad & 0.67 & $100 \%$ & $97.92 \%$ & $97.89 \%$ \\
\hline Asociación & 0.78 & $77.77 \%$ & $85.91 \%$ & $87.50 \%$ \\
\hline Bien común & 0.97 & $88.89 \%$ & $83.10 \%$ & $91.67 \%$ \\
\hline Civismo & 0.29 & $72.22 \%$ & $72.53 \%$ & $79.16 \%$ \\
\hline Colaborar & 0.35 & $88.89 \%$ & $85.92 \%$ & $87.50 \%$ \\
\hline Constitución & $0.03 *$ & $83.34 \%$ & $71.83 \%$ & $88.10 \%$ \\
\hline Conversar & 0.67 & $83.33 \%$ & $84.50 \%$ & $89.58 \%$ \\
\hline Convivencia & 0.07 & $83.33 \%$ & $89.44 \%$ & $83.33 \%$ \\
\hline Derechos & 0.91 & $94.44 \%$ & $83.80 \%$ & $83.33 \%$ \\
\hline Diálogo & 0.95 & $88.89 \%$ & $78.17 \%$ & $77.08 \%$ \\
\hline Escuchar & 0.79 & $83.33 \%$ & $85.21 \%$ & $87.50 \%$ \\
\hline Estado & 0.54 & $61.11 \%$ & $68.31 \%$ & $79.17 \%$ \\
\hline Hospitalidad & 0.39 & $77.18 \%$ & $63.31 \%$ & $95.83 \%$ \\
\hline Igualdad de Oportunidades & 0.22 & $83.33 \%$ & $85.21 \%$ & $95.83 \%$ \\
\hline Leyes & 0.93 & $66.67 \%$ & $76.06 \%$ & $75 \%$ \\
\hline Pluralismo & 0.80 & $77.78 \%$ & $76.76 \%$ & $77.08 \%$ \\
\hline Política & 0.83 & $44.44 \%$ & $38.73 \%$ & $39.58 \%$ \\
\hline Relacionarse & 0.75 & $94.44 \%$ & $92.25 \%$ & $95.83 \%$ \\
\hline Respeto & 0.68 & $88.89 \%$ & $92.25 \%$ & $97.92 \%$ \\
\hline Reunión & 0.87 & $77.78 \%$ & $78.17 \%$ & $77.08 \%$ \\
\hline Sociable & 0.80 & $77.77 \%$ & $87.32 \%$ & $83.33 \%$ \\
\hline Sociedad & 0.92 & $83.33 \%$ & $87.32 \%$ & $83.33 \%$ \\
\hline Solidario & 0.87 & $72.22 \%$ & $71.83 \%$ & $81.25 \%$ \\
\hline Tolerante & 0.32 & $77.78 \%$ & $69.72 \%$ & $60.42 \%$ \\
\hline Valor & 6.11 & $66.67 \%$ & $90.14 \%$ & $87.50 \%$ \\
\hline Nota: $*$ p $<0.05 ; * *<01$ & & & \\
\hline & & & & \\
\hline & & & \\
\hline
\end{tabular}

Por último para conocer las semejanzas encontradas en la prueba anterior, realizamos el estadístico descriptivo de tablas de contingencias, comparando cada uno de los valores sociales seleccionados con cada una de las religiones que representan la muestra. Los resultados más destacables son los siguientes: 
- Los tres grupos religiosos evaluados, coinciden en responder al valor social de amabilidad con las tasas más altas de agrado (cristianos $100 \%$, islámicos $97.92 \%$ y no creyentes $97.89 \%$ ) y al valor social de política con las tasas más bajas (cristianos $44.44 \%$, islámicos $38.73 \%$ y no creyentes $39.58 \%$ ).

- En la muestra cristiana, además del valor de amabilidad antes mencionado, son los valores de derechos y relacionarse los que han obtenido respuestas más agradables (Derecho 94.44\%, relacionarse 94.44\%). En la muestra de religión islámica, siguen al valor de amabilidad, los valores de relacionarse y respeto (relacionarse $92.25 \%$, respeto $92.25 \%$ ). Y en la muestra que no se consideran de ninguna religión, después del de amabilidad, los valores que provocan mayores tasas de agrado son el de respeto $(97.92 \%)$, seguido de los valores de relacionarse, igualdad de oportunidades y hospitalidad $(95.83 \%)$.

- Si observamos la tabla 2 vemos como todos los valores sociales evaluados, excepto el de política, tienen respuestas agradables de la muestra, sin ser la religión un factor diferenciador. Aunque no podemos olvidar, que se ha encontrado diferencias significativas en el valor social de constitución siendo el nivel de agrado en la muestra católica del $83.34 \%$, de la muestra islámica del $71.83 \%$ y de la muestra que no se considera de ninguna religión del $88.10 \%$.

\section{Discusión y conclusión}

Tras observar los resultados obtenidos, consideramos rechazar la hipótesis de partida. El alumnado que profesa diferentes religiones, no tienen respuestas diferentes a los valores sociales evaluados, excepto al valor de constitución.

Estos datos discrepan con los resultados de los estudios previos, que comparaban la influencia religiosa en la asimilación y aprendizaje de los valores. De esta forma, tanto las investigaciones que consideran que la religiosidad del individuo es un obstáculo en la adopción de valores (Bellah, 1976; Lipset, 1981; Stark, 2001), como las que reflejan conclusiones contrarias (Benson, Donahue, \& Erickson, 1989; Pérez-Delgado, 1992; Donahue \& Benson, 1995; Bridges \& Moore, 2002), difieren con nuestros resultados. Estos revelan que la religiosidad o no del alumnado no es un factor influyente en las respuestas de agrado o desagrado de los valores sociales. Tan sólo en el valor de constitución se encontraron diferencias significativas. Esta diferencia está claramente marcada en el alumnado islámico, arrojando un valor del $19.01 \%$, frente del que no profesa ninguna religión que obtuvieron un valor del $4.16 \%$. Esto puede ser debido a problemas de identidad por parte de esta fracción de la muestra. El valor constitución, puede crear la confusión de estar únicamente relacionado con el estado español. Para aquellos estudiantes islámicos cuya identidad aún no sea múltiple (Garreta, 2003) y se consideren únicamente ciudadanos marroquíes, el valor de constitución puede resultarle indiferentes y no necesario en su identidad nacional.

Aún así el hecho de que el alumnado evaluado, coincidan en considerar agradable o muy agradable todos los valores sociales presentados, es de gran relevancia. Esto puede ser una consecuencia directa de la particularidad contextual de Melilla. La ciudad autónoma lleva más de 500 años siendo intercultural. Durante todo este tiempo, ciudadanos españoles de diferentes orígenes confesionales, mayoritariamente musulmanes, cristianos, hindúes, hebreos y romaní, conviven de manera interrelacionada en un espacio no superior al de 12 kilómetros cuadrados. Esta situación intercultural puede haber facilitado la conexión e incluso el inter- 
cambio de los diferentes modos de vidas y de los valores que estos modos conllevan, tal y como afirma Buendía (2007), Ruiz (2002) y Sabariego (2002).

La ciudad de Melilla, por su trayectoria, la podemos considerar como fuente de ciudadanía intercultural (Borja \& Castells, 1997; Carneiro, 1999; Martinez, 2001; Zapata-Barrero, 2001; Cortina, 2002). Cortina (2002) define este tipo de ciudadanía como aquella que proporciona el diálogo entre culturas, el respeto a las diferencias y tiene como consecuencia la elección conjunta de valores y costumbres que merecen la pena mantener en una convivencia justa y feliz. Dicho esto, podemos pensar en vista a los resultados obtenidos, que los adolescentes melillense consideran importante mantener en la convivencia intercultural los valores sociales: amabilidad, asociación, bien común, civismo, colaborar, constitución, conversar, convivencia, derechos, diálogo, escuchar, estado, hospitalidad, igualdad de oportunidades, leyes, pluralismo, política, relacionarse, respeto, reunión, sociable, sociedad, solidario, tolerante y valor.

Por último debemos tener en cuenta las características del sistema educativo vinculado a nuestra muestra. El hecho de que a todo el alumnado le produzca una sensación de agrado todos los valores sociales presentados, puede ser una causa directa de la transmisión de valores producidos en el sistema educativo español. Los Institutos de Secundaria de la ciudad de Melilla, guían sus programas curriculares en la transmisión de valores reflejados en la constitución española y en la actual ley educativa. En dicha ley se presenta como una de los principios fundamentales, el de la transmisión y puesta en práctica de valores que favorezcan la libertad personal, la responsabilidad, la ciudadanía democrática, la solidaridad, la tolerancia, la igualdad, el respeto y la justicia, así como que ayuden a superar cualquier tipo de discriminación (LOE, 2006(BOE 106 de 4/5/2006). Art 1.c.). Todos estos valores se relacionan directamente con los valores sociales evaluados y por lo tanto el aprendizaje de los valores reflejados en la ley, puede influir en las respuestas de agrado que hemos encontrado en nuestro estudio.

No obstante, aunque los datos de la presente investigación son bastante alentadores, hay que analizarlos con la debida cautela. En primer lugar, porque la muestra del estudio no representa a la totalidad de los adolescentes melillenses, por lo que habría que ampliar el estudio a una muestra mucho más amplia. Por otro lado, porque no podemos evaluar las verdaderas causas de las diferencias significativas surgidas en nuestros resultados, por lo que tendríamos que completar esta investigación con otras, que evaluasen entre otras variables la identidad ciudadana. Finalmente, sería necesario evaluar si los resultados obtenidos en esta etapa educativa se mantienen a los largo del tiempo y si estos estudiantes, convertidos ya en adultos siguen conviviendo de forma pacífica.

A modo de resumen concluimos que:

- No existen diferencias significativas en las respuestas dadas a los valores sociales de amabilidad, asociación, bien común, civismo, colaborar, conversar, convivencia, derechos, diálogo, escuchar, estado, hospitalidad, igualdad de oportunidades, leyes, pluralismo, relacionarse, respeto, reunión, sociable, sociedad, solidario, tolerante y valor, en función de las diferentes religiones que profesan o no el alumnado. Toda la muestra evaluada, coinciden en considerar muy agradable o agradable los valores antes mencionados.

- Sólo se ha encontrado diferencias significativas en las respuestas dadas al valor social de constitución, en donde el alumnado islámico presenta tasas muy altas de indiferencia en comparación con el resto de los estudiantes evaluados. 
- En la muestra cristiana, los valores que se han considerado más agradables son amabilidad, derecho y relacionarse.

- En la muestra de religión islámica los valores que han obtenido tasas más altas de agrado son los de amabilidad, relacionarse y respeto.

- En la muestra que se considera de ninguna religión, los valores de amabilidad, relacionarse, igualdad de oportunidades y hospitalidad son los que han obtenido mayores tasas de agrado.

- Todas las religiones evaluadas coinciden en considerar al valor de política como el que menos agrado produce. 


\section{Referencias}

Batson, C. D., Oleson, K. C., Weeks, J. L., Healy, S. P., Reeves, P. J., Jennings, P. , \& Brown, T. (1979). Religious prosocial motivation: is it altruistic or egoistic? Journal of Personality and Social Psychology, 57(5), 873-884.

Beit-Hallahmi, B. (2001). Fundamentalism. In J. Krieger, the Oxford Companion to politics of the world. New York: Oxford University Press.

Bellah, R. (1976). The new religious consciousness and the crisis of modernity. In C. Glock \& R.N. Bellah (Eds.), The new religious consciousnee (pp. 133-152).

Benson, P. L. (1993). The troubled journey: a portrait of 6th-12th grade youth. Minneapolis: Search Institute.

Benson, P. L., Donahue, M. J. , \& Erickson, J. A. (1989). Adolescence and religion: a review of the literature from 1970 to 1986. Research in the Social Scientific Study of Religio, 1, 153-181.

Bernt, F. M. . (1989). Being religious and being altruistic: a study of college service volunteers. Personality and Individual Differences, 10(6), 663-669.

Bilbeny, N. (2002). Por una causa común. ética para la diversidad. Barcelona: Gedisa.

Borja, J., \& Castells, M. (1997). Local y global. La gestión de las ciudadades en la era de la información. Madrid: Taurus.

Bridges, L. J. , \& Moore, K. A. (2002). Religious involvement and children's well-being: what research tells us (and what it doesn't). Retrieved 1 october, 2002

Buendia, L. (2007). Valores interculturales para la convivencia In E Soriano (Ed.), Educación para la convivencia intercultural. Madrid: La Muralla.

Canetti-Nisim, D. (2004). The Effect of Religiosity on Endorsement of Democratic Values: The Mediating Influence of Authoritarianism. Political Behavior, 6(4), 377-398.

Carneiro, R. (1999). Proyecto Educativo de Ciudad. Educación para la ciudadanía. Paper presented at the Barcelona, por el conocimiento y la convivencia., Barcelona.

Casares, Pilar M. (1995). Test de valores: un instrumento para la evaluación. Revista Española de Pedagogía, 513-537.

Cortina, A. (2002). Ciudadanía intercultural. In J. Conill (Ed.), Glosario para una sociedad intercultural (pp. 35-42). Valencia: Bancaja.

Donahue, M. J. , \& Benson, P. L. (1995). Religion and the well-being of adolescents. Journal of Social Journal Issues, 51(2), 145-160.

Ellison, C. G. (1992). Are religious people nice people? Evidence from the National Survey of Black Americans. Social Forces, 71(2), 411-430.

Garreta, J. (2003). La integración sociocultural de las minorías étnicas (gitanos e inmigrantes). Barcelona: Anthropos.

Gearon, L., \& Brown, M. . (2003). Active participation in citizenship. In L. Gearon (Ed.), Learning to teach citizenship in the secondary school. London: Routledge.

Gervilla, E. (1991). El animador. Perfil y opciones. Madrid: CCS.

Grolnick, W. S., Deci, E. L. , \& Ryan, R. M. (1997). Internalisation within the family: the selfdetermination theory perspective. In J. E. Grusec \& L. Kuczynski (Eds.), Parenting and children's internalisation of values: a handbook of contemporary theory (pp. 135-131). New York: John Wiley \& Sons.

Grusec, J. E., \& Goodnow, J. J. (1994). Impact of parental discipline methods on the child's internalisation of values: a reconceptualization of current points of view. Developmental Psychology, $30(1), 4-19$.

Grusec, J.E. (2002). Parenting socialization and children's acquisition of values. In M. H. Bornsten (Ed.), Handbook of parenting (vol. 5): practical issues in parenting (pp. 143-167). Mahwah: Lawrence Erlbaum Associates.

Haldane, J. (1986). Religious education in a pluralist society. British Journal of Educational Studies, $34,161-181$. 
Hardy, S., Padilla-Walkera, L., \& Carlo, G. (2008). Parenting dimensions and adolescents'internalisation of moral values. Journal of Moral Education, 37(2), 205-223.

Herek, G. M. (1987). Religious orientation and prejudice: a comparison of racial and sexual attitude. Personality and Social Psychology Bulletin, 13, 34-44.

Hunsberger, B. (1995). Religion and prejudice: the role of religious fundamntalism, quest and right wing authoritarianism. Journal of Social, 51, 113-129.

Kohlberg, L. (1974). Education, moral development and faith. Journal of Moral Education, 4, 5-16.

Kohlberg, L., \& Power, C. (1981). Moral development and religious thinking and the question of seventh stage. In L. Kohlberg (Ed.), Essays on moral development (Vol. I, pp. 311-399). London: Harper and Row.

Laythe, B., Finkel, D. G., \& Kirkpatrick, L. A. . (2001). Predicting prejudice from religious fundamentalism and right-wing authoritarianism: a multiple regression approach. Journal for the scientific study of Religion, 29, 442-462.

Lipset, S.M. (1981). Political Man: the social basic of politics. Baltimore: The johns Hopkins University Press.

Ley Orgánica 2/2006, de 3 de mayo, de Educación (2006).

Martinez, V. (2001). Ciudadanos del mundo: más allá de la exclusión. Una pesperctiva desde la filosofía de la paz. In A. Cortina \& J. Conill (Eds.), Educar en la ciudadanía (pp. 233-245). Valencia: Alfons El magnámin.

Pérez-Delgado, E. (1992). Religión y moral en jóvenes adolescentes y adultos españoles. Teología Espiritual, XXXVI(108), 317-377.

Ritzema, R. J. (1979). Religiosity and altruism: faith without works?. Journal of Psychology \& Theology, $7(2), 105 .-113$.

Ruiz, C. (2002). Educación intercultural. Una visión crítica. Barcelona: Octaedro.

Sabariego, M. (2002). La educación intercultural. Antes los retos del sglo XXI. Bilbao: Desclée de Brouwer.

Schwartz, S.H., \& Huismans, S. (1995). Values priorities and religiosity in four Westerm religions. Social psychology Quarterly, 58, 88-107.

Stark, R. (2001). Reconceptualizing religion, magic, and science. Review of Religious Research, 43, 101-120.

Stouffer, S.A. (1955). Communism, Conformity, and Civil Liberties. New York: Doubleday and Co. Inc.

Sullivan, J., \& Transue, J. (1999). The psychological underpinnings of democracy: a selective review of research on political tolerance, interpersonal trust, and social capital. Annual Review of Spychology, 50, 625-650.

Wilson, J., \& Janoski, T. (1995). The contribution of religion to volunteer work. Sociology of Religion, $56(2), 137-152$.

Wylie, W., \& Forest, J. (1992). Religious fundamentalism, right-wing authoritarianism and prejudice. Psycological Reports, 71, 1291-1298.

Yik, M.S.M. , \& Tang, C.D. (1996). Linking personality and values: the importance of culturally relevant personality scale. Personality and Individual Differences, 21(5), 767-774.

Youniss, J., McLellan, J. A., \& Yates, M. (1999). Religion, community service, and identity in American youth. Journal of Adolescence, 2, 243-253.

Zapata-Barrero, R. (2001). Hacia un nuevo concepto de ciudadanía. Anthropos, 191, 3-20. 


\section{Sobre los Autores}

Dr. María Tomé Fernández: Universidad de Granada, España

Dr. Jorge Expósito Lopez: Universidad de Granada, España

Emilio Berrocal de Luna: Universidad de Granada, España 